\title{
Cuerpos, género y violencia: construcciones y deconstrucciones
}

\author{
Anna ZUROLO \\ Psicoterapeuta, Estudios de Género/ Hermes, Universidad de Nápoles Federico II \\ anna.zurolo@gmail.com \\ Francesco GARZILLO \\ Psicólogo, estudiante de doctorado en Estudios de Género de la Universidad de Nápoles \\ francesco.garzillo@libero.it
}

Recibido: 06-05-2013

Aceptado: 26-11-2013

\begin{abstract}
Resumen
Género, sexualidad e identidad son tres puntos clave de una discusión, en la que desde cualquier perspectiva que se quiera abordar no dejan de contribuir a la discusión de las y los académicos que trabajan en diversas disciplinas. Sea desde la teoría psicoanalítica, en la post-estructuralista y en la tradición filosófica de los estudios emprendidos por Butler (1990), nos encontramos con una actitud crítica frente al común binario sexo-género. En el marco de referencia, evitando así seguir la lógica del pensamiento único, abordamos el concepto de violencia basada en el género, como el conjunto de actos de abuso, acoso y otras agresiones ciegas procedentes de la necesidad de mantener cada ser humano dentro de la matriz heterosexual dicotómica, con el fin de preservar su identidad calmante y oficial. Mostramos como la violencia puede responder a un intento de aprovechar lo que está presente fuera de la "norma" y amenaza con desafiar la ley ordena toda relación interhumana.
\end{abstract}

Palabras clave: binarismo de género, violencias de género, psicoanálisis, sexualidad, heterosexismo

\section{Bodies, Gender and Violence: construction(s) and deconstruction(s)}

\begin{abstract}
The paper aims to develop a reflection on gender violence from an interdisciplinary perspective. Moving from tasks and positions of philosophical and psychoanalytic matrix , the authors bring into discussion the notion of gender based violence as a monolithic entity. After drawing a short theoretical trajectory on gender, sex and identity, the different meanings of the violence as social phenomenon are highlighted from a critical reflection on interpretative paradigms so far suggested. The main reference will be the difficulty associated with concepts such as heterosexual norm, required identity, performative gender, exclusionary effects associated with it, in order to analyze the rhetoric that act as guidance/ ordering in the speeches on gender based violence.
\end{abstract}


Key words: gender binarism, gender base violence, psycoanalysis, sexuality, heterosexim.

\section{Referencia normalizada}

Zurolo, A.; Garzillo, F.. (2013). “Cuerpos, género y violencia: construcciones y Deconstrucciones”. Política y Sociedad, Vol.50 Núm. 3 803-815

Sumario: Introducción. 1. El género entre feminismo y psicoanálisis 2. La Violencia y la violencia 3. Los cuerpos y la retórica de género en la acción. Bibliografía

No hay cuerpo sin poder y no hay poder sin cuerpos. La tecnología política del cuerpo se desplaza y se difunde, se convierte en microfísica porque está hecha por relaciones tensas y en actividad. (Trasforini 1999, 193).

\section{Introducción}

Cuerpos de géneros, cuerpos de poderes, cuerpos en contacto con violencias diariamente, actos de abuso y destrucción aptos para anular, sin conseguirlo nunca, la lógica del vínculo ${ }^{1}$.

La violencia de género es una dicción compleja, ya que ofrece una derivación inmediata a un concepto, el género, que nunca deja de inspirar el debate de los expertos que trabajan en diversas disciplinas. Es en el intento de verlo no como una entidad monolítica sino como un campo semántico muy amplio, que vamos a intentar de articular algunas coordenadas de orientación, animados por la cautela que necesariamente debe acompañar a cualquier discusión sobre la categoría de la persona humana, si es cierto que "los términos y conceptos son armas, armas de guerra" (Laplanche 2003, 74).

${ }^{1}$ Los autores de este trabajo se reconocen en su totalidad, en todas sus partes, aunque por razones de elaboración del trabajo, las aportaciones se han dividido de la siguiente manera: el epígrafe 1. El género entre feminismo y psicoanálisis, ha sido redactada por Anna Zurolo; el epígrafe 2. La Violencia y la violencia, ha sido redactada por Francesco Garzillo, y, el epígrafe 3. Cuerpos de género y retóricas en acción, ha sido escrito conjuntamente por ambos autores. 


\section{El género entre feminismo y psicoanálisis}

Tratar de debatir en psicoanálisis sobre el género es una cuestión muy compleja también en virtud de las distintas perspectivas teóricas que con los años se han venido elaborando y que dibujan otras tantas corrientes disciplinarias. De estas perspectivas, propondremos sólo algunas que más apoyan este tema.

Sexo, género, identidad, estos son los tres puntos clave de nuestra discusión/asunto/tema. Lo/las temas/cuestiones problemáticos/as desde cualquier perspectiva se los quiere definir, representan un campo conceptual de oposiciones y dicotomías: hombre-mujer, actividad-pasividad, sexo- género, naturaleza - cultura, donde los traits-d'-union parecen más bien los traits d'opposition.

A partir de ideas honestamente ambiguas de Freud - que fueron las primeras en poner en cuestión la feminidad y la masculinidad a veces como datos, otros como si fueran realidad a alcanzar-, el debate psicoanalítico siempre se ha encargado de analizar las cuestiones de género, sexo e identidad. Probablemente, sin embargo, la visión del conflicto sobre la feminidad en el psicoanálisis probablemente sea una expresión de este incesante trabajo que comenzó después de la propia producción de Freud sobre la (1924, 1931, 1932)-, ya en las décadas de los 20 y 30 del siglo pasado. Son sobre todo las reflexiones sobre la diferencia entre los sexos las que animan una disputa en los años 30, que ha visto comprometida a autores como Klein, Jones y Horney, por un lado, y a Deutsch, Bonaparte y MacBrunswick, Lampl-De-Groot por el otro (Chasseguet- Smirgel, 1964), en un intento de coger el mandato de analizar el camino por el cual la mujer se convierte en tal y repensar una teoría de la feminidad que parecía, en el camino freudiano, sacrificada.

Un primer punto de interés de la teoría freudiana está representado por la forma en que Freud parece observar, prima facie, el desarrollo de la mujer como un desarrollo similar, mutatis mutandis, al masculino. La simetría que rige estos argumentos parece alcanzar el resultado que la mujer sería nada más si no un no-varón, erosionado por sentimientos de envidia del pene y poco inclinadas a acomodar el papel "natural" de la feminidad, la maternidad.

Sin embargo, analizando escritos freudianos de la década de 1920, hay intentos de rupturas del "supuesto paralelismo" en el desarrollo psicosexual macho y hembra, de los cuales sólo brevemente proporcionamos algunos elementos. Por ejemplo, en "La disolución del complejo de Edipo" (1924) ${ }^{2}$, Freud analiza la diferente disposición del niño y la niña en relación con el descubrimiento de la castración que conduciría a una diferente extinción del complejo: en el macho la amenaza de castración, hecha realidad por la vista de los genitales femeninos, es el impulso para

${ }^{2}$ Todas las referencias introducidas han sido traducidas a efectos de la presente publicación de los textos originales en italiano e ingles, ya que este artículo fue escrito en italiano por sus autores y posteriormente traducido al español, con motivo de esta publicación. 
escapar del Edipo; en la mujer, por otro lado, el descubrimiento de la castración dará lugar a una hostilidad hacia el objeto maternal.

Sobre las peculiaridades de lo femenino, son bien conocidas las tesis freudianas sobre la oscuridad de esta dimensión: "nuestro material se convierte aquí - incomprensiblemente - en defectuoso. La hembra también desarrolla un complejo de Edipo, un súper yo y una época de latencia. ¿También se le puede asignar una organización fálica y un complejo de castración? La respuesta es afirmativa, pero la situación no puede ser idéntica a la del varón” (Freud 1924: 31-32).

Con más evidencia, la castración asume un sentido lógico y cronológicamente invertido en el hombre y en la mujer: motivo para escaparse del Edipo por un lado, impulso hacia el Edipo por la otra, en el texto del 1925, "Algunas consecuencias psíquicas de la diferencia anatómica entre los sexos”. Parece indiscutible, en última instancia, que es precisamente el tema teórico de la castración el que representa el punto más fuerte de ruptura de la simetría con la que Freud parece originariamente entender el desarrollo sexual masculino y femenino.

Siguiendo a Chasseguet-Smirgel (1964), los autores más cercanos al pensamiento freudiano como Lampl-De Groot, Deutsch y McBrunswick siguen la conceptualización freudiana que apoya la importancia de la envidia del pene como una liberación de la relación de la niña con su madre, responsable de no haberle suministrado el pene-, y también como motor del acercamiento de líbido hacia el padre. Esta agitación, en virtud de una equivalencia simbólica entre el pene y el niño, estaría en el origen del deseo femenino por la maternidad. Sin embargo, estos autores ponen de relieve cómo el deseo de tener un niño es anterior a la envidia del pene y en relación con la madre omnipotente (pre-edípica).

Por el contrario, autores que hacen referencia más directamente a la escuela psicoanalítica británica como Horney, Klein, Jones y Muller, evidencian como la envidia del pene es secundaria frente a la remoción de la vagina y de las precoces sensaciones vaginales insatisfechas y defensivas con respecto a la angustia en el interior del cuerpo, que siguen también a la primera pérdida del pecho (Chasseguet-Smirgel, 1964).

Si las primeras obras de los autores mencionados representan el principio de reflexión crítica del psicoanálisis en torno a cuestiones sobre la diferencia sexual, el momento teórico importante se halla en el trabajo de Stoller (1968), a partir del cual el discurso sobre el género comienza a tomar forma. El trabajo de Stoller aunque no sin discontinuidades teóricas y operativas, tuvo el mérito de haber propuesto al psicoanálisis estas cuestiones complejas en el intento de descifrarlas. Entre los puntos más problemáticos de la reflexión de este autor se encuentra la conocida distinción entre sexo y género, pue asigna al sexo el significado de lo que del lado masculino o femenino se refiere al cuerpo, y encuentra/coloca el género dentro de una dimensión en la frontera entre lo mental y lo cultural. Por lo tanto, el género enseña la cantidad de feminidad y masculinidad en cada sujeto. A la definición de 
núcleo de identidad de género -impregnado de proto-femineidad ${ }^{3}$ en virtud de patetismo, para ambos sexos, de la relación pre-edípica con el objeto maternal (Stoller, 1975) - corresponde la conciencia consciente e inconsciente de pertenencia a un género en lugar de otro. Esta pertenencia representa el resultado de una correlación de factores: genéticos, la atribución del sexo al nacer por parte del médico y los cuidadores, las expectativas conscientes e inconscientes de los padres en lo referente al sexo de la crianza.

Esta visión aunque vuelva a la compleja articulación de los diferentes factores involucrados en la contratación del género - biológico, cultural, relacional, psicológico - es, para otros autores, responsable de un reduccionismo excesivo. Por ejemplo, Laplanche afirma: “(...) la distinción entre sexo y género es fundamental en el psicoanálisis. Quiero darle un sentido preciso, muy diferente de las hipótesis y, en última instancia, de la confusión, introducida por Stoller. En particular, es insostenible incluir uno de los términos del lado de la anatomía y el otro del lado de la psicología. Se debería indicar con el término sexo el conjunto de las determinaciones físicas o psíquicas, comportamientos, fantasmas etcétera, conectados directamente a la función sexual y el placer sexual. Y con género el conjunto de determinaciones físicas o psíquicas, comportamientos, fantasmas, etc., vinculados a la distinción de hombres y mujeres” (Laplanche, 1980: 33)

Entonces, a partir de las confusiones generadas por esta distinción, la teoría psicoanalítica y una parte del debate post-estructuralista parecen encontrar un punto de proximidad: pues aunque ambas disiciplinas llegan a resultados diferentes comparten una actitud crítica con respecto al "código binario" sexo-género. Sin embargo, para algunas de las más conocidas posiciones psicoanalíticas sobre este tema, nos referimos en particular a la de Chiland $(1997,2002)$, una redefinición del concepto de identidad de género debe prestar más atención al lado del cuerpo, poniendo en guardia sobre el significado confuso que, cuando se refiere al lenguaje, el término induce hacia " la idea que es con el lenguaje que el niño aprende la diferencia entre sexos (Chiland 1997, 18).

Esta autora considera que la identidad sexual se constituye entre los 2 y 18 años, pero al inicio de la misma el infante no ha adquirido todavía un dominio del idioma. Su posición resulta excesivamente reduccionista porque limita el plan simbólico a una transmisión que se lleva a cabo únicamente a través del lenguaje "hablado", mientras que es posible rastrear la historia de los significados del género, y no sólo como una serie de transmisiones complejas y sutiles entre padres e hijos, que se producen a través de los sentidos, del tacto y de la vista, ya que la manera en que se acude y se habla son formas de comunicación trans-generacional (Seligman, 1998). En este sentido, parece que "la teoría psicoanalítica se interesó mas por el peso

${ }^{3}$ Stoller (1975) afirma que la primera forma de relación que el niño, de ambos sexos, vive con su madre, en un estado de indiferenciación primaria, se caracteriza por una especie de simbiosis lo que implica la asunción de los aspectos relacionados con la feminidad maternal. 
ejercido por el cuerpo sobre la representación, pero [...] es importante ver el impacto de la representación sobre el cuerpo" (Harris, 2003. 21).

En la teoría psicoanalítica (Laplanche, 1980, 2003), podemos considerar el género como una genero de asignación, dotado de requisitos de registro que afectan al ser humano que hay en el mundo, ya inmerso/sumergido en un orden simbólico. De acuerdo con esta versión, es el grupo de socios, a la venida del sujeto que prescribe, quienes inscriben el género. De esta forma, el género precede al sexo, pero sólo en la medida en que se organiza après coup ${ }^{4}$. Por lo tanto, la distinción masculino/femenino, es puramente normativa, y pertenece a un universo cultural que, creemos poder afirmar, no es lo mismo que la realidad psíquica (Napolitano y Zurolo, 2007).

Por otro lado, si nos fijamos en la filosofía, la crítica a la dualidad sexogenérucia es aún más radical. Nos referimos a la tradición académica inaugurada por Butler (1990), para quien la distinción entre sexo y género acaba siendo artificial, en la medida en que parece aludir a un orden temporal, donde el sexo, biológico, llega antes y el género instala en eso un significado cultural. Según Butler, sin embargo, la anatomía del sujeto humano no asume significado sino únicamente dentro de un orden simbólico, por lo que bajo la apariencia de la ficticia naturalidad de sí mismo, sólo hay los dos géneros socialmente construidos; siendo que la biología proporcionaría un principio de causalidad que no es más que un estratagema para apoyar la obligatoriedad de las opciones de identidad.

Por ello esta autora promueve la noción de género como un conjunto de actos performativos, es decir, el performar (Butler, 1997), como la marca de la vulnerabilidad del sujeto en el lenguaje. El género como serie repetida de actos, que se actualizan en cada momento histórico, implica una asunción por parte del sujeto de algo que confirma su pertenencia al universo masculino o femenino.

El género como performativo parece, por lo tanto, según nuestra opinión, asumir/tomar un carácter ineludible y al mismo tiempo estructural:

"(...) Este acto es un acto público. Estos actos tienen dimensiones temporales y colectivas, y su carácter público, no es sin consecuencias, el rendimiento de hecho se realiza con el objetivo estratégico de mantener el género dentro de su marco binario, un objetivo que no puede atribuirse a una sujeto, y que más bien debe entenderse como algo que se establece y consolida el sujeto"(Butler 1990, 196).

${ }^{4}$ Après coup, que en español es posterioridad, posterior, posteriormente... "Términos utilizados a menudo por Freud en relación con su concepción de la temporalidad y de las causalidades psíquicas: experiencias, impresiones y huellas de la memoria son reelaborados posteriormente a la luz de nuevas experiencias o acceso a otro nivel de desarrollo. A continuación, pueden obtener , así como una nueva forma , un nuevo eficaz psíquica” (Laplanche y Pontalis, 1967, p. 426) 
Al explicar la sujeción, se entiende que sólo viviendo y asumiendo el género se puede deconstruirlo, para que se convierta en un sitio de "protesta permanente" (Butler, 1993), esto es: un lugar de subversión política que no sólo indica la necesidad de ciertas categorías, sino la apertura hacia el embalaje/hacer y desembalaje/deshacer subjetivo.

\section{La Violencia y la violencia}

Si consideramos el género como cumbre conceptual/tema principal entonces no podemos permitirnos seguir la lógica del pensamiento único. Por lo tanto preferimos hablar de la violencia de género, tal como el conjunto de actos de abuso, de intimidaciones y en ocasiones agresión ciega procedente de la necesidad de mantener a cada sujeto humano dentro de la matriz heterosexual dicotómica, con el fin de preservar su carácter oficial y pacificador. Nos parece que la violencia, en otras palabras, puede responder a un intento de desviar lo que pasa fuera de la "norma" y amenaza con desafiar la ley que ordena cada relación interhumana.

Pero ¿de qué tipología de violencia hablamos? En Italia, la encuesta conocida ISTAT de $2006^{5}$, el informe Arcigay ${ }^{6}$, así como un creciente número de artículos, informes y estadísticas revelan un ambiente de tensión que prevalece/impregna el actual contexto sociocultural, en el que las diferencias suscitan ansiedades, miedos y angustias.

Entonces ¿Qué se entiende por violencia de género? Desde luego, no los fenómenos que se limitan al masculino / femenino. Lo que queremos destacar es que la norma de género, que requiere la identidad heterosexual como obligatoria/necesaria crea una serie de definiciones que afectan a las relaciones de poder entre los sujetos. La norma heterosexual propone un juego especular en que al masculino corresponde el femenino, y al activo le corresponde el pasivo, en una dimensión dicotómica que asigna y distribuye los poderes, y, más aún, genera efectos de exclusión de todo lo que se supone vaya en contra de esta lógica de oposición, ya sea en forma de transexual, lesbiana, homosexual o queer.

Pero esto no es suficiente. Deseamos añadir algunos ingredientes más refiriéndonos a la cuestión de la constitución de la identidad de género como una identidad melancólica, resultado de la renuncia a la adhesión homosexual "entendida [correc-

\footnotetext{
${ }^{5}$ A partir de este estudio se desprende que las mujeres entre 16 y 70 años que afirman haber sido víctimas de violencia, física o sexual por lo menos una vez en la vida son el 31,9\% de la población femenina.

${ }^{6}$ El informe recoge los episodios de la homofobia y la transfobia, que han llegado a las noticias nacionales entre 2006 y 2010, registra un total de 308 episodios de asesinatos, violación, asaltos y acoso homofóbico.
} 
ción nuestra] pasión de no vivir y la pérdida de no llorar", es decir, como objeto de forclusión (Plastina 2008, 152).

Sobre la base de las sugestiones freudianas adelantadas en Duelo y melancolía (Freud, 1915-1917), Butler señala que el padre del mismo sexo es para el niño objeto de una prohibición, una pérdida que vacila en la formación de una identidad de género tipo melancólico. La pérdida, sin embargo, no sólo implica la renuncia al objeto, sino también al deseo que hacia él, es decir, se dirige. Parece entonces que mucho antes que el tabú del incesto, motor del desarrollo psíquico y fundador del complejo de Edipo, opera/hay el tabú de la homosexualidad, en la medida en que el tabú del incesto puede entenderse como la prohibición al acceso de la unión heterosexual con el progenitor del sexo opuesto. Así para establecer los términos de las uniones heterosexuales es necesario, sin embargo, la acción de otro principio de funcionamiento: la prohibición de la homosexualidad. Como sabemos, esta reflexión es retomado por Butler de Rubin (1975): "Puesto que todas las culturas intentan reproducirse, y porque/ya que la particular identidad social del grupo familiar debe ser preservada, se establece la exogamia, y, como su presuposición, la heterosexualidad exogámica. El tabú del incesto no se limita a la prohibición de la unión sexual entre miembros de la misma línea familiar, sino también implica un tabú en contra de la homosexualidad" (Butler, 1990: 103).

Es evidente entonces que la crítica butleriana se dirige especialmente a la teoría de dos géneros opuestos y sobre todo complementarios (Butler, 2004), tesis que parece establecer la trayectoria heterosexual como obligatoria. Existen nudos relacionales que exceden la relación edipica, y desmontan la asunción de la heterosexualidad exogámica como fundamento de las relaciones humanas: "La institución de la heterosexualidad obligatoria y naturalizada requiere y regula el género como una relación binaria en la que se distingue el término masculino de lo femenino a través de las prácticas del deseo heterosexual." (Butler, 1990: 30).

La violencia de género, entonces se entendería como una expresión concreta y fáctica de la necesidad de mantener el orden de los géneros dicotómicos. En este sentido, definirla como una serie de actos de hostigamiento y abuso que tiene como sujetos a hombres y mujeres heterosexuales, implica un efecto del discurso, político, de apoyo de dicho orden.

\section{Cuerpo de género y retórica en acción}

Entonces ¿En qué consiste el acto retórico? Trasforini (1999) identifica en la violencia contra la mujer, la acción de dos distintas retóricas, una retórica del espacio y una retórica del cuerpo en situación de riesgo: el espacio de las mujeres, un espacio de capacidad limitada desde un punto de vista social y político, que reduce el margen de maniobra de las mujeres en el privado, en su interior, más seguro; y, la retórica del cuerpo en riesgo que teje el discurso médico desde los siglos XIX y XX, $\mathrm{y}$ que define al sujeto femenino como un individuo eternamente afectado por enfermedades. Ambas retóricas contribuyen a definir el espacio del cuerpo femenino 
como un espacio violable, accesible, de limites no netos. La paradója aquí es que, sin duda, la "geografía del miedo" define el espacio exterior como el espacio peligroso para la mujer, mientras que es en los espacios privados de la familia, de los hogares, donde se manifiestan muchos de los episodios de acoso y abuso contra las mujeres.

Incluso en el caso de la homosexualidad parece que funciona este doble dispositivo retórico, de manera que el cuerpo homosexual, pero también el cuerpo transgénero, es un cuerpo en riesgo, portador de infección -por ejemplo, pensemos en las campañas de prevención del SIDA ${ }^{7}$-, donde el espacio exterior, espacio que se basa en la necesidad de hacer visible (fuera) la opción sexual, acaba teniendo efectos de la domesticación y asimilación de los individuos menos peligrosos. Como es sabido, si la revelación representa una encrucijada existencial para cada persona gay, lesbiana, bisexual o transexual, vista como liberación, no siempre es ese el caso en la escena pública. La única imagen de la "diversidad sexual" que puede tener espacio es de hecho extremadamente camp (Satong, 1998) ${ }^{8}$, una imagen que se conforma con cuerpos delgados, afeminados, maquillados, irreconocibles. A través de esta significación del cuerpo, el elemento inquietante del "diferente” está desactivado, actualizándolo y reafirmando el valor absoluto de la dicotomía masculino-femenino (Dall'Orto, 1990).

Lo que está en juego es un dispositivo de poder, que básicamente apunta al cuerpo. El cuerpo de la mujer, el cuerpo del homosexual, del transexual; cuerpos, que son, en última instancia, cuerpos de género:

"Los estudios sobre la violencia a menudo ignoran el propio cuerpo, perdiendo de vista que se trata de cuerpos de géneros, en los que los significados se han establecido y están continuamente cuestionados, construidos y fabricados por (y sobre) los lenguajes y prácticas que se relacionan con la violencia, con su percepción, con el miedo, todos los días. De hecho, no existe un cuerpo neutral, excluido del proceso continuo de producción cultural de significado, al igual que no hay un cuerpo que espera pasivamente el desciframiento objetivo de los expertos, en cambio existen un vocabulario y sintaxis del cuerpo que, como los de todos los idiomas, son culturalmente proporcionados " (Trasforini, 1999: 193).

¿A qué nos referimos cuando pensando en la violencia, ponemos el cierpo en el centro? Un punto interesante de reflexión es ofrecido por la filósofa Nussbaum $(2004,2010)$, que rastrea en la base de la discriminación y la violencia, la emoción

${ }^{7}$ Un análisis de cómo se ha construido este aspecto es parte de este monográfico., a cargo de F. Villaamil.

${ }^{8}$ La autora define el campo como la capacidad de interpretar la realidad únicamente dentro de la estética exagerada, teatral. En este sentido, nos referimos al término camp como una actitud en la que se amplian dramáticamente los códigos de género dejando espacio a una interpretación teatral pura de la norma cultural de género binario. 
primaria y visceral de repugnancia que implican las reacciones físicas hacia estímulos que a menudo tienen características corporales. El asco - en la obra de Nussbaum- tiene que ver con la relación problemática que tenemos con nuestra mortalidad, con la descomposición de la carne y con los aspectos fundamentales de lo que define a cada ser humano, "generalmente se refiere a un grupo de objetos primarios: heces, sangre, semen, orina, moco, sangre menstrual [...]" (Nussbaum, 2010: 85) Estos son los objetos primarios que están asociados y proyectados hacia el otro, lo que implica un doble efecto: la fantasía sobre los otros como corruptos por la suciedad del cuerpo y la tranquilidad en nuestra propia pureza.

Como señala esta autora, no resulta sorprendente que el disgusto aparezcca con frecuencia en el contexto de la sexualidad que consiste en el intercambio de líquidos y sustancias orgánicas, lo que nos califica como seres corpóreos y no como seres trascendentes. Lo que inspira repugnancia es típicamente la fantasía que los hombres tienen hacia el varón homosexual como analmente penetrable. La idea de semen y las heces que se mezclan dentro del cuerpo de un hombre es fuertemente repugnante para aquellos que consideran la no penetrabilidad un límite sagrado que protege contra la suciedad y la muerte. La homofobia -observa Nussbaum-, está estrictamente vinculada a la misoginia: en los clásicos locus de la repugnancia contra un grupo está el cuerpo femenino (Nussbaum 2004, 139). Las mujeres reciben la semilla y se creen contaminadas por esta substancia y juzgadas así, por ello, como criaturas tan débiles, inestables, con partículas contaminantes e impuras: "Puesto que la mujer recibe la semilla por el hombre, ella se convierte en su desagradable parte mortal, de la cual el hombre siente la necesidad de distanciarse" (Nussbaum 2004: 140).

Entonces, misoginia y homofobía tienen la misma matriz: la preocupación compartida por los fluidos corporales que encuentra expresión en la estigmatización de quienes lo reciben y quedan sometidas a una condición de medio salvaje:

"Todas las sociedades, al parecer, definen algunos seres humanos como asquerosos. Muy probablemente es una farsa para proteger más firmemente el grupo dominante por el miedo de su propia animalidad: si estos humanoides se encuentran entre yo y el mundo de la animalidad desagradable, estoy más lejos de ser mortal/ decadente/ oloroso/ baboso" (Nussbaum, 2010: 86).

Aunque existen profundas diferencias entre los resultados prácticos de las teorías de Butler y Nussbam ${ }^{9}$, creemos que la contribución de esta última da valor "corporal" a la estrategia de abyección que se identifica con el trabajo de Butler. Esta estrategia, constitutiva del dispositivo heterosexista, estabiliza las posiciones sexuales que bordean el masculino y el femenino en comparación con un estándar que, por un lado, permite identificarse con uno de los dos sexos dictados por el simbolismo,

\footnotetext{
${ }^{9}$ Para más detalles, ver Nussbaum (2009).
} 
mientras, por otro lado, la regla como tal, excluye a las personas que no pertenecen, produciendo al excluido -lo abyecto-, ese ser asqueroso que amenaza a la normalidad que es la humanidad

Deseamos seguir ahondando en la estrategia de la abyección con algún ingrediente más. La abyección, del latín abjícere ( $a b$, por y jàcere, tirar) se refiere a tirar, para cazar por sí mismo, y se engancha al concepto psicoanalítico de repudio (Verwerfung) que consiste en la expulsión de los contenidos psíquicos fuera del sujeto. Para Lacan, la forclusión define lo que está fuera de la simbolización, ya que fue rechazado por la psique, y que no ha dejado huella, pero que vuelve en el mundo real (Kaës, 2009). En este punto, seguimos a a Kristeva (1980) con el fin de comprender mejor lo que significa la abyección y cuáles son los dispositivos de exclusión del sujeto. En esta autora, lo abyecto se refiere a la primaria remoción, no es sujeto ni objeto, pues del objeto se recupera sólo la calidad de oponerse al yo, super yo, y se ha exiliado fuera: "Y, sin embargo, este exilio de la miseria no deja de desafiar a su amo. [...] A cada uno su objeto, a cada super yo, su abyecto" (Kristeva, 1980: 4).

Volvemos aquí a las muestras abyectas como la orina, las heces, el semen y la sangre, o sea a las sustancias que debilitan la división entre interior y exterior, sujeto y objeto, y que debe ser rechazada por la narración del Yo. Esta extrañeza, la abyección, que era familiar y que se rechazó como repugnante y asquerosa, es la valla y el injerto del sujeto, que pone de relieve las limitaciones y la abyección de sí mismo, que es prueba de que la degradación es también el reconocimiento de la ausencia como un elemento fundamental de todos y cada uno de los sujetos:

"Si bien es cierto que lo abyecto insta al sujeto y si bien lo pulveriza, se entiende cómo la persona se sentiría en su fuerza máxima en cuanto, cansado de vanos intentos para reconocerse fuera de si, descubre lo imposible en sí mismo: cuando encuentra que lo imposible es su mismo ser, descubriendo ser sólo abyecto. La abyección de sí sería la forma culminante de la experiencia de la persona a la que se revela que todos sus objetos se basan simplemente en la pérdida inaugural que es la base de su bienestar. Nada más que el abyección de si mismo muestra que cada abyección es el reconocimiento de la falta fundador de cualquier ser, el sentido, el idioma, el deseo" (Kristeva 1980, 7).

Entonces, las justificaciones médicas, que hemos mencionado al principio de esta sección, son únicamente intentos de fuga que convierten en abyecto lo que perturba la identidad, el sistema, el orden; aquello que no respeta los límites, los lugares, las reglas. "El intermedio, lo ambiguo, lo mixto" (Kristeva, 1980: 7). Por ello, consideramos que a fin de plantar las fronteras de los lugares fantásticos de normalidad -el macho, la heterosexualidad y la pureza- se necesita de alguien que claramente amenace la norma.

En la amenaza a la norma, Butler (1993), encuentra también una fractura dentro de la propia norma que se cita a sí misma, capaz de redefinir nuevas y más fluidas identidades que rompan los términos de hombre y mujer del simbolismo normal, es decir, el paradigma heterosexual dominante. Influenciada por las teorías de Foucault $(1975,1976)$ que promete ser un espacio de resistencia y subversión de los 
cuerpos con respecto a la norma, en lugar de una declaración de quiebra en contra de un orden simbólico permanente, Butler afrma que las prácticas queer realizan una "conversión de la abyección en acción política" (1996, 20), abriendo paso a una política que da legitimidad a los cuerpos excluidos del cuerpo social, a los cuerpos abyectos.

\section{Nota:}

Esta publicación ha sido producida con el financiamiento de HERMES European Project (2011-2013) del Daphne III Programme, Comisión Europea. El contenido de esta publicación es de sola responsabilidad del autor y no refleja necesariamente la posición de la Comisión Europea.

\section{Bibliografía}

Butler, J. (1990): Scambi di genere. Identità, sesso e desiderio, Milano: Sansoni, 2004.

Butler J. (1993): Corpi che contano. I limiti discorsivi del sesso, Milano: Feltrinelli, 1996

Butler J. (1997): Excitable speech. A politics of the performative,. New York: Routledge.

Butler J. (2004): La disfatta del genere. Roma: Meltemi, 2006.

Chasseguet-Smirgel J. (1964) La sessualità femminile. Nuove ricerche psicoanalitiche. Bari: Laterza 1995.

Chiland C. (1997) : Changer de sexe. Paris: Odile Jacob.

Chiland C. (2002): "Gender and sexual difference”, en Dialogues on sexuality, gender and psycho-analysis, 79-91. London: Karnac Books.

Dall’Orto, G. (1990): “Mediterranean homosexuality”, en Wayne R. Dynes (a cura di) Encyclopedia of homosexuality, 2: 796-798.

Foucault, M. (1975): Sorvegliare e punire, Torino: Einaudi, 1976.

Foucault, M. (1976): La volontà di sapere. Storia della sessualità 1, Milano: Feltrinelli, 1978.

Freud, S. (1915-1917): Lutto e melanconia. OSF VIII, Torino: Bollati Boringhieri, 1989

Freud, S. (1924): Il tramonto del complesso edipico. OSF X. Torino: Bollati Boringhieri, 1989.

Freud, S. (1925): Alcune conseguenze psichiche della differenza anatomica tra i sessi. OSF X. Torino: Bollati Boringhieri, 1989.

Freud S. (1931): Sessualità femminile. OSF XI, Torino: Bollati Boringhieri, 1989.

Freud S. (1932): Introduzione alla psicoanalisi. Lezione 33. La femminilità. OSF XI. Torino: Bollati Boringhieri, 1989. 
Harris, A. (2003): Identità di genere: un concetto "in restauro”. Ricerca Psicoanalitica, XIV - 1): 7 - 28

Kaës, R. (2009): Le alleanze inconsce. Roma: Borla 2010

Kristeva J. (1980): Poteri dell’orrore. Saggio sull'abiezione. Milano: Spirali, 2006

Laplanche, J. y Pontalis, J.-B. (1967): Enciclopedia della psicoanalisi. Bari: Laterza, 1993.

Laplanche, J. (1980): Problematiche II. Castrazione. Simbolizzazioni. Bari-Roma: La Biblioteca, 2000.

Laplanche, J. y Gubrich-Simitis, I. (2003) : Sur la théorie de la séduction. Libres cahiers pour la psychanalyse. Paris: In Press Éditions.

Napolitano S. y Zurolo A. (2007) "Questioni di genere. Riflessioni tra poststrutturalismo, decostruzionismo e psicoanalisi”, Psicoterapia e scienze umane, XLI - 2: 190-204.

Nussbaum, M. C. (2010): Disgusto e umanità. L'orientamento sessuale di fronte alla legg,. Milano: Il Saggiatore, 2011.

Nussbaum, M. C. (2009): The Professor of Parody. Retrieved March, 01, 2013 from http://www.akad.se/Nussbaum.pdf

Nussbaum, M. C. (2004): Nascondere l'umanità. Il disgusto, la vergogna, la legge. Roma: Carrocci, 2007

Palombi, F. (2008): "Un intermedio surreale. L'aporia di Antigone tra Judith Butler e Jacques Lacan”, e M. Pasquino y S. Plastina S. (a cura di): Fare e disfare. Otto saggi a partire da Judith Butler, 123-138Milano-Udine: Mimesis,.

Plastina, S. (2008): "L'enigma della melanconia. Identità e rappresentazione della vita psichica in J. Butler. En Pasquino M. \& Plastina S. (a cura di). Fare e disfare. Otto saggi a partire da Judith Butler., 139-154, Milano-Udine: Mimesis,.

Rubin, G. (1975) "The traffic in women: notes on the "political economy" of sex", en Reiter R. (Ed.):. Toward an Anthropology of Women, 157-209, New York: Monthly Review Press.

Satong ,S. (1998): Note su «camp», in Contro l'interpretazione, Milano: Mondadori

Seligman, S. (1998): "Child psychoanalysis, adult psychoanalysis and developmental psychology: introduction”, Psychoanalytic Dialogues, 8: 1, 79-86.

Stoller, R. J. (1968): Sex and gender. The development of masculinity and femininity, New York-London: Karnac Books, 1974.

Stoller, R. J. (1975a): Perversion. The erotic form of hatred, London: Karnac, 1986.

Stoller, R. J. (1975b): The Transsexual Experiment (Sex and Gender volume II), London: The Hogarth Press and The Institute of Psycho-Analysis.

Trasforini, M.A. (1999): "Il corpo accessibile. Una riflessione su corpi di genere, violenza e spazio”, Polis, 2: 191-212. 